\title{
Korpuslinguistik, Hermeneutik und die soziale Konstruktion der Wirklichkeit
}

\author{
Wolfgang Teubert (Birmingham)
}

\begin{abstract}
In this article, I attempt to embed corpus linguistics in a framework of social constructionism and hermeneutics. Corpus linguistics, as I see it, has a unique approach to meaning. It aims to describe the meaning of a unit under consideration exclusively through usage and paraphrase. These units can be seen as objects of the discourse, and their meaning is what has been said about them within the discourse. While over the last forty years corpora were mainly used to find out what is common to different occurrences of such units, for instance a lexical item such as friendly fire, we should now turn our attention also to what make each occurrence a singular event, somehow building on paraphrases before the event and the traces this particular event leaves in subsequent texts. I am not interested in what is going on in the minds of speakers and hearers, and I am not interested in how what is said may refer to reality. Meaning as seen here is only found within the discourse, negotiated in a never-ending flow of contributions to the discourse, in which what has been said so far is continuously rearranged in and permuted in new contributions to the discourse. The discourse is therefore autopoietic and self-referential.
\end{abstract}

\section{Die Gesellschaft setzt Sprache voraus, und umgekehrt}

Die Gesellschaft kann, im Sinne von Niklas Luhmann (Luhmann 1998), als eine Struktur verstanden werden, die das betrifft, was zwischen Menschen, autonomen, über Intentionalität, d.h. Bewusstsein verfügenden Subjekten, stattfindet. Damit ist impliziert, dass die Menschen selber und ihr Bewusstsein nicht Teil der so verstandenen Gesellschaft sind. Ihr Bewusstsein, ihre Intentionalität, ihre Ich-Erlebnisse, ihre Intentionen und ihr Verstehen sind der Gesellschaft nicht direkt zugänglich. Nur das, was die Mitglieder der Gesellschaft als Zeugnis ihres Verstehens zum Diskurs beitragen, gehört zum Gegenstandsbereich von Gesellschaft. Diese Beiträge sind verbale Äusserungen, Texte also, und sie machen den Diskurs einer Gesellschaft aus. Die Struktur einer Gesellschaft manifestiert sich also in ihrem Diskurs, d.h. in der Gesamtheit aller Äusserungen einer Gesellschaft, verstanden als Diskursgemeinschaft. Damit ist, aus meiner Sicht, auch die soziale Praxis nur insoweit Teil der Gesellschaftsstruktur, als sie zum Objekt des Diskurses wird, als über sie gesprochen wird. Eine soziale Praxis, die nicht zum Gegenstand des Diskurses gemacht wird, ist auch nicht Teil der diskursiv konstruierten Wirklichkeit. Dies ist meine zentrale Kritik am Programm der Kritischen Diskursanaly- 
se (critical discourse analysis; CDA), wie es vor allem von Norman Fairclough (Fairclough 2003) und Ruth Wodak (Wodak 1988) formuliert wird. Dort wird der Diskurs der diskursexternen sozialen Praxis gegenübergestellt.

Nur Menschen organisieren sich als Gesellschaft. Dazu bedürfen sie der Sprache. Denn nur Sprache macht es möglich, soziale Praxis als soziale Praxis zu begreifen. Geburtstage, Ausflüge, Zusammenarbeit, Arbeitsteilung, Demonstrationen und Feiern sind abstrakte Konstrukte, und deshalb bedürfen sie des sprachlichen Ausdrucks, um als solche verstanden zu werden. Ohne ein Wort für Arbeit gäbe es Arbeit nicht als Begriff; ebensowenig gäbe es Eigentum oder Recht, oder auch die Gesellschaft als solche. Denn nur ein Mensch kann sich bewusst sein, Mitglied einer Gesellschaft zu sein. Er ist sich nur deshalb dessen bewusst, weil die Gesellschaft etwas ist, worüber gesprochen wird.

Eine menschliche Gesellschaft kann es nur geben, wo es Sprache gibt. Andererseits bedarf es der menschlichen Gesellschaft, um die Fähigkeit zu erlernen, zu sprechen, eine Äusserung zu tun, mit anderen Mitgliedern der Gesellschaft zu kommunizieren, Inhalte auszutauschen und zu teilen. Menschen, die ausserhalb der Gesellschaft, in Isolation von anderen Menschen aufwachsen, lernen nicht zu sprechen. Sie können deshalb kein autonomes Bewusstsein entwickeln.

\section{$2 \quad$ Unsere Wirklichkeit ist ein Konstrukt des Diskurses}

Sprache ist kein Spiegel der Wirklichkeit. Sprache referiert nicht auf die Wirklichkeit. Sprache als Diskurs ist immer nur autoreferentiell. Sie referiert auf das, was schon gesagt ist.

Wie also steht es um die Verbindung von Sprache und Wirklichkeit? Ursprünglich war Sprache immer gesprochene Sprache. Doch ist Sprache in oralen Gesellschaften in der Regel kein Gegenstand des Diskurses. Sie ist eine soziale Praxis, derer man sich zumeist nicht bewusst ist. In oralen Gesellschaften spricht man nicht über Sprache. Wie es scheint, verfügen viele schriftlose Sprachen nicht über ein Wort für das Diskursobjekt Wort (Goody 1987). In oralen Gesellschaften interessiert nicht, was eine Äusserung bedeutet. Vielmehr geht es darum, was der Sprecher meint, um dessen Absichten. Sprechen und Hören sind simultan. Sie sind situativ eingebettet in das, was sich in der Gruppe abspielt, in das, was sich im Umkreis des Geschehens befindet. Mündliche Sprache ist voller Deixis. Es gibt Demonstrativ-, Personal- und Possessivpronomen, es gibt Orts- und Zeitadverbien, es gibt Gestik, Mimik und Intonation (Ricoeur 1981). Das Bezeugen von Ich-Erlebnissen durch den Sprecher und das Ich-Erlebnis des Hörers sind nicht voneinander getrennt. Die diskursexterne Wirklichkeit liegt in Sichtweite dessen, was gesagt wird. Die Sprache oraler Gesellschaften ist konkret. Die Fähigkeit zur Abstraktion ist kaum entwickelt. Selbst einfache Syllogismen werden nicht verstanden. Intelligenztests, wie wir sie kennen, wären unanwendbar. Es würde keinen Sinn machen, über abstrakte Zusammenhänge zu reflektieren. Mangels einer Möglichkeit, den Inhalt von Reflexion aufzubewahren und später einmal weiter zu entwickeln, erweist sich jede Reflexion als verlorene Liebesmühe. Selbst die Konfrontation mit fremden Sprachen führt, so scheint es, nicht zu einer Reflektion über Sprache: Man bezeichnet Menschen, die man nicht versteht, als Barbaren, als Fremde, Welsche, oder als Stumme, also als Menschen ausserhalb 
der Gesellschaft. Orale Gesellschaften kennen keine Geschichte, keine Schulen, keine Philosophie und keine Linguistik (Ong 2002, Luria 1976).

In einer oralen Gesellschaft kann der Gedanke nicht aufkommen, dass Wörter Zeichen sind, die für etwas anderes stehen. Ein Zeichen hat stets Materialität; während die gesprochene Sprache immateriell ist. Der Gedanke, dass eine Äusserung eine von der Intention des Sprechers unabhängige Bedeutung hat, kann in einer oralen Gesellschaft nicht gedacht werden. Dass Sprache als symbolisches System verstanden wird, setzt Schriftlichkeit voraus. Weil das Diskursobjekt Wort fehlt, kann in oralen Gesellschaften auch metaphorisches Sprechen nicht als solches erkannt werden. Denn wenn Bedeutung nur als Sprecherintention verstanden wird, ist sie dialogischer Aushandlung entzogen.

Gesprochene Sprache lebt von Deixis. Was der Sprecher meint, muss konkretisierbar sein, muss dem Hörer als erinnertes Ich-Erlebnis zur Verfügung stehen. Diskursobjekte, die nicht erlebbar sind, sind nur als Mythen, die immer gleich vorgetragen werden, denkbar. Dass sie immer gleich erzählt werden können, ist nur möglich, wenn die Inhalte für den Erzähler Sinn machen, das heisst mit seinen Ich-Erlebnissen verknüpft werden können. Eine Gesellschaft ohne Schrift verfügt nur sehr beschränkt über ein kollektives Gedächtnis. Anstelle des Diskursobjekts Geschichte, verbunden mit der Vorstellung von Entwicklung, gibt es nur die geschichtslose Gegenwart des immer Gleichen, verbunden mit den Kreisläufen von Mondphasen, Jahren und Generationen. Anstelle von Geschichte gibt es zeitlose Mythen. Der Diskurs in einer oralen Gesellschaft hat keine diachronische Dimension. Etwas Gesagtes kann nicht mit dem verglichen werden, was früher gesagt worden ist. Allenfalls ist es möglich, etwas Gesagtes mit zeitlosen Merksprüchen zu vergleichen.

Mit der Schrift verliert sich die Unmittelbarkeit von Rede. Das Geschehen des Sprechens findet nicht mehr statt. Am Akt des Schreibens hat der Leser keinen Anteil. Die unmittelbare Beziehung zwischen dem Gesagten und der diskursexternen Wirklichkeit ist verloren. Ohne Deixis, ohne Ostentation bezieht sich Sprache nicht mehr auf die Aussenwelt, sondern nur noch auf sich selber. An die Stelle der Wirklichkeit tritt der Diskurs, die Gesamtheit all dessen, was zwischen den Mitgliedern einer Gesellschaft an verbalen Äusserungen ausgetauscht worden ist und ausgetauscht wird. Das was geschrieben wird, referiert auf Aussagen, die man selber oder die andere früher gemacht haben. Das schafft eine der mündlichen Rede fremde Distanz, die nur durch einen besonderen und bewussten Akt der Aneignung zu überwinden ist (Ricoeur 1981). Wenn ich verstehen will, was da geschrieben steht, muss ich es in Ich-Erlebnisse umsetzen, beispielsweise indem ich es mit einer selbst erlebten Situation abgleiche oder mich an früher Gelesenes erinnere. Jede Abstraktion vergrössert meine Distanz zur Wirklichkeit. Womit ich in einer literalen Gesellschaft sprachlich operiere, bezieht sich nicht mehr direkt auf die Wirklichkeit, sondern konstituiert sich in Abstraktionen, die immer schon die Wirklichkeit interpretieren und mich dadurch von der Wirklichkeit entfremden. Der Diskurs schafft sich seine eigenen Objekte. Diese Diskursobjekte sind Konstrukte wie Familie, Kindheit, Religion, Schule, Nation, Depression, Schönheit, Eigentum, Arbeit, Sprache usw. Auch wenn Diskursobjekte als gesellschaftliche Institutionen realisiert werden, bleiben diese Institutionen Konstrukte des Diskurses und beziehen ihre Bedeutung ausschliesslich aus 
dem Diskurs (Berger/Luckmann 1980). Der Diskurs ist autoreferentiell. Im Reden über die Diskursobjekte wird dialogisch ausgehandelt, was sie bedeuten.

Anders als der kognitiven Linguistik geht es der Korpuslinguistik nicht darum, zu verstehen, was der Sprecher will, wie wir also von einer sprachlichen Äusserung auf die Intentionen des Sprechers schliessen können. Die Diskursteilnehmer, die Mitglieder einer Diskursgemeinschaft mit ihren jeweiligen Intentionalitäten, entziehen sich dem Zugriff der Korpuslinguistik. Nur der Diskurs, verstanden als die vergangene und gegenwärtige Kommunikation zwischen den Diskursteilnehmern, ist ihr Gegenstandsbereich. Ein neuer Text, der in den Diskurs eingebracht wird, wird nicht als bewusste geistige Schöpfung, sondern als Rekombination und Permutation bereits existierender Texte verstanden. Der Diskurs hat somit immer eine diachronische Dimension. Ein Text, auf den in nachfolgenden Texten referiert wird, wirkt mit an der diskursiven Konstruktion der Wirklichkeit. Was er bedeutet, lässt sich an den Spuren ablesen, die er hinterlässt (Luhmann 1998). Die Wirklichkeit, die im Diskurs konstruiert wird, ist nicht homogen, und sie ist nicht stabil. Aber abgesehen von unserer eigenen eng umgrenzten Lebenswelt ist die Wirklichkeit des Diskurses die einzige Wirklichkeit, die uns zugänglich ist (Watzlawick 1977).

Der Diskurs ist die Konkretisierung von Sprache. Er ist, im Sinne von Ferdinand de Saussure, parole. Während la langue, das Sprachsystem, notwendig spekulativ bleibt, ist der Diskurs empirisch. Sprache, die Fähigkeit, einen Beitrag zum Diskurs zu leisten, ist das, was Gesellschaft möglich macht. Deshalb verfügen nur Menschen über Gesellschaft.

\section{Die Korpuslinguistik beschäftigt sich nur mit archivierter Sprache}

Sprachwissenschaft setzt geschriebene oder sonstwie festgehaltene Sprache voraus, Sprache, die, wie auch immer, archiviert ist und wieder zugänglich gemacht werden kann. Gesprochene Sprache, die im Augenblick ihres Entstehens bereits vergeht, ist einer Analyse unzugänglich. Auch wenn die Linguistik gesprochene Sprache untersucht, ist sie auf Transkriptionen, wenigstens auf Tonaufnahmen angewiesen. Denn sonst hat sie keine Daten, an denen wissenschaftliche Behauptungen gemessen werden können. Jede Wissenschaft, auch die Linguistik, setzt Gesellschaft voraus, eine Gemeinschaft von Experten, die durch ihren eigenen Diskurs verbunden sind (Foucault 1969). Alle Diskursbeiträge, alle wissenschaftlichen Analysen, gelten nur im Rahmen einer dialogischen Aushandlung. Ein wissenschaftlicher Text muss belegen, was er behauptet. Er muss auf überprüfbaren Daten basieren.

Die Intentionalität von Sprecher und Hörer bleibt dem wissenschaftlichen Zugriff entzogen. Der Versuch, sie in ein Modell des Geistes zu integrieren, müsste, wie Daniel Dennett und John Searle unabhängig voneinander plausibel gemacht haben, zu einem infiniten Regress führen (Dennett 1991, Searle 1992). Im Sinne Luhmanns (Luhmann 1998) kann und muss der Diskurs ('Kommunikation' bei Luhmann) von Sprechern und Hörern als systematisch dissoziiert betrachtet werden. Doch im Ereignis gesprochener Sprache sind Sprecher, Hörer, Situation und soziale Strukturen untrennbar miteinander verwoben. Wenn wir in einem Gespräch etwas nicht verstehen, fragen wir: 'Was hast du gemeint?'. Wir befragen den Sprecher nach seiner Intention. Im Falle geschriebener Sprache fragen wir statt dessen: 'Was 
bedeutet das Geschriebene?' Der Text, die Äusserung ist disloziert vom Sprecher. Die Intentionen des Autors sind irrelevant dafür, was der Text bedeutet. Die (korpus-)linguistische Analyse des Diskurses klammert den Sprecher und seine Intentionalität aus. Dazu sagt Paul Ricoeur: "Inscription becomes synonymous with the semantic autonomy of the text, which results from the disconnection of the mental intention of the author from the verbal meaning of the text, of what the author meant and what the text means. What the text means now matters more than what the author meant when he wrote it." (Ricoeur 1976).

\section{Die Abwesenheit des Sprechers erzeugt die Illusion von Wahrheit}

Anders als in gesprochener Sprache stehen sich in geschriebenen Texten Sprecher und Hörer nicht gegenüber.

Sprecher können lügen. Was sie sagen, bezieht sich auf die Sprechern und Hörern potentiell präsente Wirklichkeit. Ich erkenne eine Lüge daran, dass das Gesagte nicht mit meinem IchErlebnis übereinstimmt. Lügen setzen jemanden der lügt voraus: den Sprecher. Dagegen sind die Autoren von geschriebenen Texten dem Leser nicht verfügbar. Menschen können lügen; Texte lügen nicht. Sie haben eine Bedeutung. Das macht die Faszination von geschriebenen Texten aus. Die Gleichsetzung von Bedeutung und Referenz (in Freges Terminologie: von Sinn und Bedeutung) verleiht geschriebenen Texten die Illusion von Wahrheit und, verbunden damit, Heiligkeit. Das erklärt die Attraktivität des Ausspruchs "denn es steht geschrieben ...". Davon machen Religionen gern Gebrauch:

For it stands written: Worship the Lord your God, and serve Him only. daily.presbycan.ca/devotions/1997/97-02-25.html

For it stands written in the law: the sun should not set on one that has been put to death www.mithraism.erudition.net/append/passage $3 . \mathrm{htm}$

For it stands written in the Kitvei HaKodesh, HINENI YISSAD B'TZION EVEN PINNAT YIKRAT www.beittikvahsynagogue.org/obh/engtexts/1skefa.htm

For it is written, in Surah Al-Maa'idah 5:116, 'And behold, Allah will say - O Jesus Son of Mary, did you say to men, worship me and my mother as gods beside www.theonion.com/onion3513/christ_islam.html - 23k - 9 May 2003

Je weniger sich Texte an unseren Ich-Erlebnissen messen können, desto wahrer erscheinen sie. Je unverständlicher ein Text ist, desto tiefer wähnt man gern die in ihm enthaltene Wahrheit. So lesen wir bei Rousseau: "Dans les trois révélations, les livres sacrés sont écrits en des langues inconnues aux peuples qui les suivent. Les Juifs n'entendent plus l'hébreu, les Chrétiens n'entendent ni l'hébreu ni le grec; les Turcs ni les Persans n'entendent point l'arabe; et les Arabes modernes eux-mêmes ne parlent plus la langue de Mahomet. Ne voilàt-il pas une manière bien simple d'instruire les hommes, de leur parler toujours une langue qu'ils n'entendent point?" (Rousseau: Emile §1074). 


\section{$5 \quad$ Die Bedeutung ist im Diskurs}

Von Saussure haben wir gelernt, dass das sprachliche Zeichen, für Saussure in der Regel das Wort, für mich der Text oder ein Textsegment, unter zwei Aspekten zu sehen ist: Form und Bedeutung. Anders herum: Nur das was Form und Bedeutung hat, kann als Text (-segment) analysiert werden. Der Text bedeutet. Was heisst es dann, wenn wir nach der Bedeutung eines Texts fragen? Was wir erhoffen, ist nicht eine Tautologie, sondern eine Paraphrase, etwas, was uns den Text als Ganzes oder ein Textsegment, etwa eine feste Fügung wie friendly fire, erläutert. Die Fügung friendly fire ist zugleich Form und Bedeutung. Doch nicht jedes Mitglied der Diskursgemeinschaft muss diese Fügung und seine Bedeutung kennen. Der Novize benötigt eine Erklärung, eine Erläuterung, eine Umschreibung, eine Paraphrase dieses Diskursobjekts. Der Begriff Diskursobjekt erscheint mir nützlich, weil er die Unterscheidung zwischen Wortwissen und Weltwissen aufhebt. Eine solche Unterscheidung mag sinnvoll sein, wo wir an Sprache als einem strukturalistisch zu erfassendem System interessiert sind. Wenn wir indessen Sprache als parole, als Diskurs beschreiben, erübrigt sich die Trennung. Wenn ich erkläre, was friendly fire ist, habe ich auch erklärt, was friendly fire bedeutet. Das unterscheidet ja gerade natürliche Sprachen von formalen Sprachen (wie die Sprache der Mathematik oder Logik oder wie Programmiersprachen), dass natürliche Sprache autoreferentiell ist, dass man mit Sprache über Sprache spricht, dass also Paraphrasen nicht einer gesonderten Metasprache angehören, sondern der Sprache selbst. Nur die Pedanterie des 19. Jahrhunderts verführt uns dazu, mit Gänsefüsschen und Kursivschrift die Ebenen zu unterscheiden, wie beispielsweise im zweiten dieser (der Mannheimer Korpussammlung entnommenen) Korpusbelege:

Und fast schon ans Zynische grenzt jene Bezeichnung, welche die Militärsprache für den irrtümlichen Beschuss der eigenen Leute kennt. Man nennt das friendly fire - freundliches Feuer. An dieser Frontlinie beobachten wir auch immer wieder das, was die Militaristen "friendly fire" nennen, nämlich Verluste in den eigenen Reihen durch fehlgeleitete Geschosse aus den eigenen, nachfolgenden Linien. Was die Haider-Diskussion anlangt, hat sich dieses Phänomen sogar zu einer Art intellektueller Selbstschussanlage verfestigt.

Das Diskursobjekt wird paraphrasiert, und so werden wir seiner Bedeutung gewärtig. Wir mögen glauben, dass wir die Bedeutung des Wortes Löwe kennen, weil man uns Löwen in freier Wildbahn oder im Zoo gezeigt hat. Aber keiner hat uns je ein Einhorn zeigen können, und doch ist uns dieses Diskursobjekt ebenso geläufig. Liegt es daran, dass man uns Bilder von Einhörnern gezeigt hat? Aber woher wissen die Maler oder Zeichner, wie sie aussehen? Am Ursprung kann nur eine Paraphrase gestanden haben. Auch erschöpft sich das, was wir über konkrete Dinge wie Löwen wissen, keineswegs in deren Aussehen. Wir wissen auch, das Löwen die Könige der Tierwelt sind und dass sie mutig wie eben nur Löwen sind, dass sie in Rudeln leben und dass sie zu den Katzenartigen gehören, Eigenschaften, die sich über ihr Aussehen nicht erschliessen.

Was also sprachliche Zeichen, Wörter, Fügungen, Textsegmente, ja ganze Texte bedeuten, erfahren wir aus dem Diskurs. In der Tat werden wir so mit neuen Diskursobjekten vertraut. Wir wissen, was Globalisierung ist, weil man es uns unablässig mitteilt. Google listet über 
24000 Vorkommen von "Globalisierung bedeutet". Die ersten acht Belege (abgefragt am 17.04.06) sind:

Globalisierung bedeutet die weltumspannende Ausdehnung kapitalistischer Wirtschaft,

Globalisierung bedeutet, dass Nationalstaaten mit allen anderen Nationalstaaten konkurrieren und eine protektionistische Politik betreiben

Globalisierung bedeutet, dass es auf der ganzen Welt die gleichen Bürger zu

Der Ausdruck Globalisierung bedeutet in Wirklichkeit Amerikanisierung.

"Globalisierung bedeutet Amerikanisierung"

Globalisierung bedeutet, dass der Welthandel und die Finanzmärkte stärker integriert werden

Globalisierung bedeutet, dass die Grosskapitalisten nicht mehr nur die Leute in ...

Globalisierung bedeutet nicht nur das Streben nach höheren Umsätzen durch den Verkauf von Produkten und Dienstleistungen auf den globalen Märkten.

Alles, was über ein Diskursobjekt gesagt wird, ist Teil seiner Bedeutung. Natürlich kann niemand die 24000 Paraphrasen kennen, die Google findet, und noch weniger die unzähligen anderen, die je Eingang in den Diskurs gefunden haben. Jeder Diskursteilnehmer wird daher seine eigene Auffassung von der Bedeutung haben, auf der Grundlage der Paraphrasen, mit denen er vertraut ist. Jeden Tag gibt es zudem neue Paraphrasen, und mit jeder neuen Paraphrase, sofern sie nicht schlicht wiederholt, was bereits gesagt ist, ändert sich die Bedeutung. Wie also können wir uns überhaupt verständigen?

\section{$6 \quad$ Was ist der Diskurs? Was repräsentiert ein Korpus?}

Der Diskurs, habe ich eingangs gesagt, ist die Gesamtheit aller Äusserungen einer Diskursgemeinschaft. Was aber ist die Diskursgemeinschaft? Im weitesten Sinn ist sie die Gemeinschaft aller, die mit ihren sprachlichen Äusserungen zum Gesamtdiskurs der Menschheit beitragen. All das, was nicht aufgeschrieben oder sonstwie festgehalten wird, verschwindet im Augenblick seiner Entstehung und entzieht sich so dem Zugriff der Linguistik. Aber auch das, was geschrieben ist, ist zu amorph und zu gigantisch, als dass es sich erfassen liesse. Auch dieser Gesamtdiskurs, also das, was an sprachlichen Äusserungen zwischen allen Menschen dieser Erde ausgetauscht und (schriftlich) festgehalten wird, kann in seiner Gesamtheit nicht analysiert werden. Eine Beschränkung auf einzelne Diskurse ist notwendig. Diese Diskurse existieren nicht schlechthin; vielmehr sind es sprachwisssenschaftliche (oder sozialwissenschaftliche) Konstrukte. Es ist der Forscher, der den Diskurs, den er untersuchen will, definieren, begründen und gegenüber der Gemeinschaft der Forscher rechtfertigen muss. Dazu muss er Parameter festlegen, beispielsweise eine bestimmte Sprache, einen zeitlichen Ausschnitt, das Vorhandensein in gedruckter oder elektronischer Form, eine bestimmte Auflagenhöhe oder Leserschaft, ein bestimmtes Genre, eine bestimmte Stilebene oder inhaltliche Kriterien (Teubert 2004).

Diskurse sind sprachwissenschaftliche Konstrukte (Busse/Teubert 1994). Ist unser Ziel etwa die lexikographische Beschreibung des deutschen Wortschatzes, müssen wir den Diskurs 
entsprechend definieren. So beschränken wir uns auf das Deutsche als Sprache und vielleicht auf einen sehr engen Zeitrahmen wie etwa die letzten fünfzig Jahre. Wir können ferner festlegen, dass wir nur mit gedruckten Quellen arbeiten, und dass diese Quellen in elektronischer Form zur Verfügung stehen müssen. Aber es wäre immer noch eine Sisyphusaufgabe, diesen Diskurs verfügbar zu machen. Der finanzielle und manuelle Aufwand wäre unabsehbar und unbezahlbar. Machbar ist lediglich eine überschaubare Stichprobe des Diskurses. Das Korpus, die empirische Basis der Korpuslinguistik, ist eine solche Stichprobe.

Das erste grosse Projekt der Korpuslinguistik war das Cobuild-Wörterbuchprojekt, das 1980 vom britischen Wörterbuchverlag Collins gemeinsam mit der Universität Birmingham in Angriff genommen wurde. Leiter war John Sinclair. Die erste Auflage dieses ersten einsprachigen korpusbasierten Wörterbuchs erschien 1987. Es verzeichnete nur Wörter, die im Korpus enthalten waren und es arrangierte ihre Bedeutungen nach der Häufigkeit, mit der sie im Korpus vertreten waren. Allerdings wurden die Bedeutungserklärungen (das, was ich oben Paraphrasen genannt habe) nicht dem Korpus entnommen, sondern vom Lexikographenteam auf der Grundlage der Korpusbelege erarbeitet. Einer der Gründe dafür ist, dass das Korpus, das dem Wörterbuch zunächst zugrunde lag, nur eine Umfang von sieben Millionen Wörtern hatte, und erst im Lauf der Zeit vergrössert wurde, bis es vor einigen Jahren (als Bank of English) auf knapp eine halbe Milliarde angewachsen ist (Krishnamuthy 1992).

Wie gross muss ein Korpus also sein, das ausreichend ist für ein Standardwörterbuch? Darauf gibt es keine vernünftige Antwort. Das Mannheimer Institut für Deutsche Sprache verfügt inzwischen über Korpusressourcen von ca. drei Milliarden Wörtern. Es ist eine Sammlung von Korpora, die ganz unterschiedliche Diskurse repräsentieren, nicht ein homogenes Korpus. Ob es für ein Wörterbuch ausreicht, hängt davon ab, welchen Wortschatz und welchen Diskurs das Wörterbuch darstellen soll. Wie ich oben am Beispiel Globalisierung gezeigt habe, können wir heute auch das Internet als Korpus verwenden. Aber es würde uns schwer fallen, den Diskurs zu beschreiben, den das Internet repräsentiert.

\section{$7 \quad$ Was will die Korpuslinguistik?}

Korpuslinguistik ist empirisch. Ihr Gegenstand sind echte (d.h. nicht erfundene) Sprachdaten: Texte oder Textteile, die nach explizit gemachten Prinzipien und Kriterien zu einem Korpus zusammengestellt sind. Die Korpuslinguistik macht allgemeine und spezifische Aussagen über den Diskurs. Im Vordergrund der Korpusarbeit der vergangenen vierzig Jahre stand das Streben nach Feststellungen über sprachliche Phänomene, die sich aufgrund von Häufigkeit und statistisch beobachtbarer Signifikanz verallgemeinern lassen. Was die Lexik angeht, so ging es primär darum, lexikalische Einheiten zu erkennen. Anders als viele Wörter sind lexikalische Einheiten monosem. Sie bestehen oft aus mehreren Wörtern, die im Korpus signifikant häufig miteinander vorkommen und sich so mit entsprechen statistischen Verfahren ermitteln lassen. Die statistische Analyse allein ist jedoch nicht ausreichend. Dazu muss die semantische Relevanz kommen. Sie liegt vor, wenn alle Vorkommen einer lexikalischen Einheit dieselbe Bedeutung teilen. Die Bedeutung komplexer lexikalischer Einheiten lässt sich also nicht als Addition der Bedeutungen der Wörter, aus der sie bestehen, auffassen. 
Vielmehr hat jede lexikalische Einheit ihre eigene Bedeutung (s.o. das Beispiel friendly fire). Sobald wir nicht mehr das Einzelwort in seiner Isolation im Wörterbuch, sondern die lexikalische Einheit, wie wir sie im Korpus ermitteln können, in den Mittelpunkt unserer lexikalischen Untersuchung stellen, löst sich das Problem der semantischen Ambiguität, das die traditionell Linguistik so lange beschäftigt hat, in Nichts auf. Das Wort ist keine 'natürliche' Einheit; es ist ein linguistisches Konstrukt, das sich in semantischer Hinsicht trotz seines ehrwürdigen Alters von über tausend Jahren nicht bewährt hat, wie praktisch es immer für die Analyse der Morphosyntax flektierender indoeuropäischer Sprachen sein mag (Halliday/Teubert/Yallop 2004).

Das Konstrukt der 'lexikalischen Einheit' als Bedeutungseinheit ist der Korpuslinguistik zu verdanken. Heutzutage stehen Kollokationen im Zentrum lexikalischer Analyse. Ohne die Korpuslinguistik könnten wir ihrer nicht gewahr werden.

Jedesmal wenn eine lexikalische Einheit in einem Korpus vorkommt, können wir dies als die Vorkommen, als ein 'token' eines 'types' einer lexikalischen Einheit sehen. Der 'type' ist also eine Generalisierung, eine Verallgemeinerung, die aus dem besteht, was allen Vorkommen, allen 'tokens' gemeinsam ist. Wenn wir also eine lexikalische Einheit ansetzen, postulieren wir damit einen Gemeinsamkeitsanspruch. Dabei müssen wir uns im Klaren sein, dass die lexikalische Einheit so wenig wie das Wort eine 'natürliche' Existenz hat. Lexikalische Einheiten erweisen sich lediglich als sinnvolle Konstrukte, wenn es um die Beschreibung von Bedeutung geht.

Verallgemeinerung setzt voraus, dass wir jedem Vorkommen eines Phänomentyps dieselbe Relevanz zuweisen. Wir sind nicht daran interessiert, was ein Vorkommen von friendly fire von einem anderen unterscheidet, sondern nur daran, was ihnen gemeinsam ist. Das setzt eine synchronische Betrachtungsweise voraus, eine Betrachtungsweise, in der jedem Vorkommen derselbe Wert zukommt. Dies war in den zurückliegenden vierzig Jahren der Schwerpunkt korpuslinguistischer Arbeit.

\section{Bedeutungsbeschreibung in synchronischer Betrachtungsweise}

Die Korpuslinguistik hat sprachwissenschaftliche Traditionen revolutioniert. An die Stelle der Analyse einzelner willkürlich ausgesuchter Beispiele tritt die Analyse aller einschlägigen Belege im Korpus. Das macht Zusammenhänge sichtbar, die früher höchstens zufällig entdeckt wurden. Was die lexikographische Bedeutungsbeschreibung angeht, betrifft das, wie oben angedeutet, in erster Linie das Kollokationsverhalten von Wörtern. Die Methodik der Korpuslinguistik zeigt auf, dass Wörter keineswegs beliebig kookkurrieren, sondern dass es relativ feste statistische Verhältnisse für das Miteinandervorkommen von Wörtern gibt. Wenn wir diese lexikalischen Muster erkennen, löst sich die Polysemie, die Ambiguität auf, die ein Resultat traditioneller Betrachtungsweise ist. Wie ein Blick in herkömmliche Wörterbücher zeigt, haben vor allem häufigere Wörter eine Vielzahl von 'Bedeutungen'. Indessen ist dieser Befund kontrainduktiv, denn beim Lesen eines Textes sind wir uns dieser Polysemie in den seltensten Fällen bewusst. Der Kontext legt fest, welche Lesart eines Wortes zu wählen ist. In Verbindung mit seinen Kollokaten löst sich die Mehrdeutigkeit eines Einzelworts auf. Wort 
und Kollokat bilden eine monoseme Bedeutungseinheit, eine Kollokation. Ich zeige dies hier am Beispiel des Adjektivs arm. Dieses Adjektiv hat nach dem Deutschen Universalwörterbuch (1983) drei Bedeutungen: a) ohne [genügend] Geld zum Leben, wenig besitzend, bedürftig, mittellos, b) wenig habend, aufweisend od. hergebend, ohne nutzbringenden Gehalt, ärmlich, c) unglücklich, bedauernswert, beklagenswert. Wenn wir die Adjektiv-SubstantivKollokationen (extrahiert als statistisch signifikant und relativ häufig aus einem den Mannheimer Korpora entnommenen Spezialkorpus von ca. 50 Millionen Wörtern) betrachten, ist diese Polysemie in den meisten Fällen nicht gegenwärtig. Wir finden (in Klammern: Häufigkeit; Bedeutung; [Bemerkung]; Beleg): []

armes Ding (10; c); metaphorisch oder feste Fügung; das arme Ding hat nichts zu lachen)

armes Geschöpf (9; c); metaphorisch[?]; das arme Geschöpf kommt zu mir)

armer Hund (11; c); [fast immer] feste Fügung; er war ein armer Hund wie ich)

armer Kerl (19; c); feste Fügung?; er ist gestürzt wie dieser arme Kerl)

armes Kind (30; c); was hat man dir, armes Kind, getan?)

arme Kirchenmaus (5; b[?]; [fast immer] feste Fügung; die sprichwörtliche arme Kirchenmaus)

arme Länder (25; a); es war eines der ärmsten Länder)

armes Mädchen (25; a), b) oder c); [oft ]feste Fügung; ein armes Mädchen aus dem Volk; das arme Mädchen kann einem leid tun)

armer Narr (5; c); feste Fügung; Armer Narr! dachte er)

armer Schlucker (9; a) oder b); feste Fügung; es hat aus ihnen arme Schlucker gemacht)

arme Seele (23; c); feste Fügung; hinter ihnen her wie der Teufel hinter der armen Seele)

armer Sünder (6; c); feste Fügung; ich bin ein armer Sünder)

armer Teufel (21; c); [fast immer] feste Fügung; die armen Teufel sehen erbärmlich aus)

armes Tier (13; c); wir werden das arme Tier vor dem Opfertod retten)

armer Tropf (5; c), [selten a)]; feste Fügung; gestorben ist der arme Tropf)

armer Vater (28; c); mein armer Vater war ein Gebrochener)

In fast allen Fällen gibt es nur eine Lesart für die aus arm und einem Substantiv bestehende Kollokation. Im Fall von armes Mädchen hängt die Lesart vom weiteren Kontext ab. Manche Fragen bleiben offen: Was macht eine feste Fügung aus? Handelt es sich bei Ding in armes Ding um eine Metapher oder ist armes Ding eine feste Fügung? Metaphern und feste Fügungen sind Diskursobjekte, sind sprachwissenschaftliche Konstrukte. Sie referieren nicht auf metaphysische Realitäten. Es ist die Aufgabe des Wissenschaftlers, sie so zu definieren, dass ihm andere Wissenschaftler zustimmen. Weitaus interessanter ist die Frage, ob ein armer Tropf mittellos, ärmlich oder bedauernswert ist. Sie zeigt zum einen, dass feste Fügungen nicht leicht in die Teile zerlegt werden können, aus denen sie bestehen, zum anderen, dass oft nicht möglich ist, die Bedeutungsangaben des Wörterbuchs auf einen Textbeleg abzubilden. Denn diese Bedeutungsangaben gaukeln vor, disjunktiv zu sein, wo nichts als Unschärfe, fuzziness, vorliegt. Wer wenig besitzt, hat auch wenig, und deshalb ist er bedauernswert. 
Das Beispiel hat, so hoffe ich gezeigt, dass das Einzelwort in der Isolation des Wörterbuchs uns nur sehr unvollkommen zeigen kann, was das Wort, eingebettet in Text und Kontext, bedeutet. Eine kleine Auswahl aus den Belegen für "ein armer Tropf ist", die google.de am 21.04.06 verzeichnet hat, ist da viel aufschlussreicher:

Ein Milliardär ohne eigenes Museum ist ein armer Tropf.

Was für ein armer Tropf ist doch der reichste Knopf ohne eine Geschenkidee!

Ein armer Tropf ist der, der keine Phantasie hat

..., dass der Hauptcharakter so ein armer Tropf ist, macht ihn total sympathisch!

Nur weil der ein armer Tropf ist, der von seinem Land in den Krieg geschickt wurde?

Ich bin in der Tat der Meinung, dass derjenige ein armer Tropf ist, der noch nie "Fear and Loathing in Las Vegas" gesehn hat

ein armer Tropf ist jener, der beim schreiben oder lesen nichts empfinden kann

ein individualist ist ein armer tropf

\section{Die diachronische Dimension des Diskurses macht Intertextualität sichtbar}

Soweit wir an Verallgemeinerung und der Feststellung von dem, was Einzelfällen gemeinsam ist, interessiert sind, sind wir auf eine synchronische Betrachtungsweise angewiesen. Die Möglichkeit, über statistische Signifikanz Kookkurrenzen in den Griff zu bekommen, hat lange die Faszination von Korpuslinguistik ausgemacht. Inzwischen ist die Methodik zur Analyse von Häufigkeiten gut entwickelt. Ein breit gefächerter Werkzeugkasten von Computerprogrammen steht dafür zur Verfügung. Häufigkeitsuntersuchungen sind wesentliche Hilfsmittel, wenn es um Bedeutung geht. Doch deckt die synchronische Perspektive allein das Phänomen Bedeutung nicht ab. Komplementär dazu gehört die diachronische Perspektive (Teubert 2005).

Die Texte, die den Diskurs ausmachen, werden nicht simultan in den Diskurs eingebracht. Wenn immer ein neuer Beitrag gemacht wird, können wir ihn als Reaktion auf das, was bereits gesagt ist, sehen. Jeder neue Text wiederholt in grossen Teilen bereits Gesagtes. Textsegmente, Kollokationen, lexikalische Einheiten, die bereits früher Eingang in den Diskurs gefunden haben, werden rekombiniert, permutiert, in neue Kontext eingebaut, und auf neue Weise paraphrasiert. Ein neuer Text nimmt auf, akzeptiert, modifiziert, reinterpretiert oder weist zurück, was in anderen Texten enthalten ist. Jedes Vorkommen von friendly fire hat Aspekte, die wir auch in allen anderen Vorkommen von friendly fire finden. Aber wenn der Text nicht komplett wiederholt, was in früheren Texten gesagt ist, hat jedes neue Vorkommen auch singuläre Aspekte, Aspekte, die es von allen anderen Vorkommen unterscheiden.

Wir können den Diskurs also auch als ein Nacheinander von Textbeiträgen sehen. Frühere Texte hinterlassen Spuren in späteren Texten; spätere Texte verweisen auf frühere Texte, manchmal explizit, häufig implizit. Was die Texte eines Diskurses miteinander verbindet, ist das Phänomen der Intertextualität (Allan 2000). Dabei geht es nicht darum, ob den Sprechern 
oder Hörern solche intertextuellen Bezüge bewusst sind. Die Korpuslinguistik untersucht Korpora, als Stichproben von Diskursen; an dem, was in den Köpfen der Mitglieder einer Diskursgemeinschaft vor sich geht, ist sie nicht interessiert.

Deutlich wird das Phänomen der Intertextualität, wenn wir Paraphrasen untersuchen. Wenn jemand sagt, was eine lexikalische Einheit, ein Diskursobjekt wie Globalisierung bedeutet, tut er das in aller Regel auf dem Hintergrund von dem, was in anderen Diskursbeiträgen bereits gesagt ist. Wir wissen inzwischen, dass das Wort globalization in seiner heutigen Bedeutung zuerst 1969 in der Harvard Business Review verwendet wurde (Teubert 2002). Nicht jeder Text, der Globalisierung paraphrasiert, bezieht sich direkt auf diesen Erstbeleg. Aber indirekt ist der Bezug vorhanden, ganz gleich, ob er dem Verfasser des Texts bewusst ist oder nicht (Kristeva 1986).

Eine Untersuchung von Intertextualität kann Ähnlichkeiten zwischen Belegen feststellen, aber auch den markierten Widerspruch zu früheren Äusserungen. Weil solche Ähnlichkeiten zufällig sein können, ist es sinnvoll, nach weiteren Gemeinsamkeiten zwischen den Texten, denen die jeweiligen Belege entstammen, zu suchen. Wenn man etwa die Texte, die man etwa auf einer Webpage britischer Euroskeptiker findet, miteinander abgleicht, kann man davon ausgehen, dass sie sich mit einer gewissen Wahrscheinlichkeit aufeinander beziehen oder wenigstens auf denselben Text oder dieselben Texte, die den britischen Euroskeptizismus begründet haben, wie beispielsweise Maggie Thatchers berühmte Rede, die sie in Brügge am 20. September 1988 gehalten hat. Dort sagte sie unter anderem:

Europe will be stronger precisely because it has France as France, Spain as Spain, Britain as Britain, each with its own customs, traditions and identity. It would be folly to try to fit them into some sort of identikit European personality. (meine Hervorhebung)

Die Fügung identikit European war bis zum Zeitpunkt ihrer Rede nicht belegt. Sie ist ungewöhnlich, und es wäre statistisch gesehen äusserst unwahrscheinlich, wenngleich nicht unmöglich, dass sie ein zweites Mal erfunden würde. Google verzeichnet (am 18.04.06) 86 Belege. Dazu kommen 57000 Belege für das gemeinsame Vorkommen der beiden Wörter im selben Text. Viele dieser Belege sind offensichtliche Zitate; andere benutzen die Fügung in neuen Kontexten, in denen sie gelegentlich dazu dient, den Text im Spektrum des Euroskeptizismus zu verorten, oder den Euroskeptizismus zu kritisieren, oft aber auch einfach nur, um eine Aussage zu ironisieren. Beispiele sind:

Who is trying to create some sort of Identikit European personality, who are your real targets in that speech?

Gone were Cardiff's decaying docks, replaced by identikit European-style waterfront developments

There is no "identikit" model for structural reform of European economies

The identikit of European recession is completed by the impoverishment of families

while the remaining cast reads like an identikit of European cinema 
Wenn wir unsere Google-Suche um mehr Wörter aus der Thatcher-Rede erweitern, nämlich um traditions, customs und identity, finden wir einen wachsenden Anteil an Texten, die dem britischen Euroskeptizismus zuzurechnen sind:

Our identity as a nation is migrating to the European Union and we are left empty as a result. ... its own customs, traditions and identity.

I would see it as a net loss actually in the European tradition, ... in a sort of identikit way, in order to try and create a pseudo-European identity, ...

superiority and the desire to protect traditions are just as easily held by those on the ... reducing British identity to "some sort of identikit European ...

After all the crowing about the superiority of the European social model in the ... undermine age old traditions and even the intrinsic identity of a nation ...

embrace oral traditions, customs, languages, ... We might also stray into the European debate as it affects how we feel about losing our identity to a ...

Wir können also das Phänomen Intertextualität auch dazu benutzen, um Texte zusammen zu gruppieren, um einen Spezialdiskurs zu konstruieren, einen Diskurs also, der aus Texten besteht, die sich in bestimmter Weise aufeinander beziehen. Intertextualität ist ferner wichtig, wenn es darum geht, die Relevanz von Texten innerhalb eines Diskurses zu bewerten. Texte, die mehr Spuren in nachfolgenden Texten hinterlassen, sind relevanter als solche, auf die sich nachfolgende Texte nicht oder nur in geringem Mass beziehen. Intertextualität ist sicherlich ein Parameter, der wichtiger ist als etwa Auflagenhöhe oder Leserschaft.

\section{Die Bedeutungstheorie der diachronischen Korpuslinguistik ist die Hermeneutik}

Der diachronischen Korpuslinguistik, wie ich sie verstehe, geht es darum, herauszufinden, was ein Text oder ein Textsegment oder eine lexikalische Einheit zu einem gegebenen Zeitpunkt bedeutet. Dabei ist zunächst zu klären, was Bedeutung in diesem Zusammenhang ist. Die Bedeutung eines Textes oder eines Textsegments ist seine von der Diskursgemeinschaft (oder Teilen von ihr) anerkannte Interpretation. Damit ist klar, dass eine Interpretation ein Text ist, ein sprachlicher Beitrag zum Diskurs. Eine erfolgreiche Interpretation ist eine, die Spuren in nachfolgenden Interpretationen hinterlässt. Daraus ergibt sich, dass die Handlung des Interpretierens grundsätzlich dialogisch ist. Eine Interpretation ist stets eine Interpretation für andere (Teubert 2003).

Dem entgegengesetzt ist das Verstehen eines Texts oder Textsegments. Wenn ich ein Gedicht verstehe, bewirkt es in mir ein Ich-Erlebnis. Dieses Ich-Erlebnis ist für andere nicht direkt zugänglich, es ist als solches nicht vermittelbar. Nie kann ich einem anderen hinreichend beschreiben, was dieses Gedicht für mich bedeutet. Nur indem ich dieses Ich-Erlebnis in ein sprachliches Zeugnis überführe, also es in einen Text umwandle und diesen Text in den Diskurs einbringe, kann ich vom Verstehen zur Interpretation gelangen. Das Verstehen ist ein individueller mentaler Akt; die Interpretation ist ein kollektiver Akt, an dem weitere Diskursteilnehmer als (zumindest imaginäre) Rezipienten beteiligt sind. 
Beim Interpretieren eines Textes oder eines Textsegments geht es nicht, wie Schleiermacher behauptet hat, darum, diesen Text besser als sein Autor zu verstehen. Die Autorschaft eines Texts ist ein soziales Konstrukt. Ob das Ich, das aus dem Text spricht, mit dem Ich des Autors identisch ist, ist irrelevant. Der Autor steht dem Interpreten in der Regel nicht als Dialogpartner gegenüber. Was er über den Text zu sagen hätte, wäre ebenfalls nur eine Interpretation, ein Beitrag zur Diskussion, der den gleichen Stellenwert hätte wie jede andere.

Eine Interpretation eines Texts oder Textsegments ist eine Paraphrase, aber zugleich auch mehr als eine Paraphrase. Zur Interpretation des Satzes "There is no "identikit" model for structural reform of European economies" gehört, dass die Wörter identikit und European auf Maggie Thatcher verweisen und sie indirekt da kritisieren, wo sie selbst ein Modell, nämlich das britische, als Modell für ganz Europa ansieht, während die andererseits mit der Fügung identikit Europe ein einheitliches (nicht-britisches) Modell für die Struktur Europas ablehnt. Eine hermeneutische Interpretation bettet den zu interpretierenden Text in einen Diskurs ein und identifiziert die intertextuellen Bezüge.

Handelt es sich um einen älteren Text, bei dem wir nicht sicher sein können, ob die lexikalischen Einheiten, aus denen er besteht, einen Bedeutungswandel durchgemacht haben, wird die Aufgabe der hermeneutischen Interpretation noch komplexer. Denn es geht dann nicht mehr nur darum, den Text in einen relevanten Diskurs von Texten, die ihre Spuren hinterlassen haben, einzubetten, sondern auch darum, unser jetziges Textverständnis mit der Weise, in der er zur Zeit seiner Entstehung gelesen wurde, in Übereinstimmung zu bringen. Es geht also um die "Verschmelzung zweier Horizonte", wie Hans Georg Gadamer das genannt hat. Im Prinzip ist es unmöglich, einen älteren Text so zu lesen, als wäre der Diskurs seither nicht weitergekommen. Vergleichbar wäre das mit dem vergeblichen Versuch, eine Komposition von Bach so zu hören, als habe es seither keinen Beethoven, Schubert oder Schostakovitsch gegeben. Was bleibt, ist das Bemühen, uns bewusst zu machen, was sich seither verändert hat. Wir müssen die Texte, die auf den zu interpretierenden Text Bezug nehmen, daraufhin lesen, was sie zur Interpretation beitragen. Eine Horizontverschmelzung im Gadamerschen Sinn bedeutet, dass wir das, was der Text seinerzeit bedeutet, in Verbindung bringen mit dem, was er uns heute bedeutet (Gadamer 1965). Die Interpretation eines (älteren) Textes kann sich also nicht darin erschöpfen, was der Text zur Zeit seiner Genese bedeutet hat. Es geht immer auch darum, was er jetzt bedeutet. Jede Bezugnahme auf den zu interpretierenden Text, die seither stattgefunden hat, leistet einen Beitrag zur Bedeutung des Textes heute. Das Ergebnis des Versuchs, die seinerzeitige Bedeutung eines Texts mit seiner heutigen Bedeutung zu verschmelzen, kann damit immer nur eine Annäherung sein.

Gadamer ist insoweit der Romantik verhaftet geblieben, als er glaubt, die Bedeutung eines Textes liesse sich nicht durch die Anwendung einer wissenschaftlichen Methode, sondern letztlich nur durch Empathie erschliessen. Was das Textverstehen angeht, ist das unabweisbar. Aber gilt es auch für die Interpretation als einen kollektiven dialogischen Akt? Wer einen Text interpretiert, tut dies nicht nur für sich selber, sondern er wagt sich mit dem Akt der Verbalisierung, der Bezeugung seines Ich-Erlebnisses immer auch an eine Öffentlichkeit (die letztlich auch imaginär oder virtuell sein kann). Es mag nicht um Wahrheit gehen, aber doch 
um die Akzeptanz, die sich in den Spuren äussert, die diese Interpretation wieder in nachfolgenden Texten hinterlässt.

Woran liegt die Akzeptanz einer Interpretation? Die Indizien, die zu überzeugen vermögen, sind zum einen Paraphrasen, zum andern intertextuelle Bezüge, durch die sich die Paraphrasen in anderen Texten auf den zu interpretierenden Text beziehen lassen. Was etwa Maggie Thatchers Fügung 'identikit European personality' heute bedeutet, lassen die Paraphrasen ermessen, die Google als Ergebnis der Anfrage thatcher identikit european aufwirft, beispielsweise:

By 1989 Mrs Thatcher had become vociferously 'anti-European', ... of the identities of the European peoples, to be replaced by an "identikit-European".

Thatcher started out pro EU in 1979, but she didn't like the way it evolved. ... on the populations of European countries and they are forced into identikit ...

reducing British identity to "some sort of identikit European personality" (Geddes 2004, 196). Thatcher's fear of what she recognized as a socialist ...

Identikit Europeans, as Mrs Thatcher warned us against in her Bruges speech to the College of Europe? Does 'cultural cohesion' imply a 'Fortress Europe' ...

The Thatcher nightmare of an Identikit Europe is becoming all too real . Germans skive off work and linger laughingly round pools ; British males commandeer ...

In der Tat hat die Korpuslinguistik Verfahren entwickelt, mit denen man Paraphrasen und ähnliche Textsegmente finden kann. Was indessen nicht operationalisierbar ist, ist die Definition des Diskurses, innerhalb dessen sich eine Interpretation zu bewegen hat. Das sind Entscheidungen, die sich erläutern lassen, die aber stets subjektiv bleiben. Insofern kann die Korpuslinguistik einen wichtigen Beitrag dazu leisten, sich dialogisch über die Bedeutung eines Textes oder Textsegments zu verständigen. Doch eine Methodik im Sinne Gadamers kann es nicht geben.

Der hermeneutische Bedeutungsansatz, wie ich ihn hier vorschlage, hat zur Konsequenz, dass sich die Bedeutung eines Texts oder Textsegments nie abschliessend ausgehandelt werden kann. Jede neue Interpretation verändert, soweit sie Neues sagt und soweit sie in nachfolgenden Texten Spuren hinterlässt, die Bedeutung. Eine Bedeutungsanalyse aus diachronischer Perspektive kann immer nur vorläufig sein.

\section{$11 \quad$ Wer legt die Bedeutungen fest?}

Es ist immer die Diskursgemeinschaft, die die Bedeutung von lexikalischen Einheiten aushandelt. Der Diskurs ist, wie ich oben ausgeführt habe, autoreferentiell. Wir benutzen Sprache, um über Sprache zu sprechen. Es ist die Sprache, die es ermöglicht, neue Objekte in den Diskurs einzuführen oder alte Diskursobjekte wieder der Vergangenheit zu entreissen. Kaum wird in Berlin die Tochter einer islamischen Familie von ihrem Bruder ermordet, weil sie sich von ihrem Mann getrennt hat, beschäftigt sich der deutsche Mediendiskurs mit dem Diskurskonstrukt der Zwangsehe. Das Wort ist nicht neu; ausweislich des Grimmschen Wörterbuchs hat es bereits Jean Paul verwendet: "...dass Neigungsehen so schlecht und Zwangsehen 
oft gut ausfallen". Was wir heute unter dem Konzept der Zwangsehe zu verstehen haben, wird vom Mediendiskurs diktiert, gemäss der News-Funktion von Google.de am 18.04.06:

Wolfgang Bosbach spricht sich dafür aus, Zwangsehen in Deutschland unter Strafe zu stellen.

Allerdings können Zwangsehen schon jetzt mit bis zu fünf Jahren Haft bestraft werden.

Zwangsehen sind in Europa keine Randerscheinung,

Die religiös oder kulturell begründete Tradition von Zwangsehen soll per Gesetz unterbunden werden.

Er nannte Zwangsehen "eine Menschenrechtsverletzung, die strafrechtlich und ausländerrechtlich" geahndet werden müsse.

Neben Zwangsehen und weiblicher Genitalverstümmelung gehören auch Ehrenmorde zur sogenannten traditionsbedingten Gewalt.

Zwangsehen sind ebenso abzulehnen wie Schwimmunterricht-Dispensen.

Der familienpolitische Sprecher der Unions-Fraktion, Johannes Singhammer, sagte, Zwangsehen seien keine private oder kulturell tolerierbare Angelegenheit.

Die Belege zeigen, dass das, was ich Paraphrase nenne, nicht etwas ist, was wir als solches im Text finden, sondern dass es eine spezielle Perspektive der Analyse ist. Man könnte dieselben Belege auch unter der Perspektive Sprachgebrauch betrachten: 'Wie wird das Wort Zwangsehe verwendet?' Unter der Perspektive von Paraphrase geht es mir darum, festzustellen, was über das gegebene Diskursobjekt ausgesagt wird. Paraphrasen differenzieren nicht, wie bereits oben gesagt, zwischen 'Wortwissen' und 'Sachwissen' Es geht hier nicht um den Versuch einer strukturalistischen Beschreibung im Sinne von genus proximum und differentia specifica. Das, was ich als Paraphrase analysiere, lässt sich gut mit dem Pierceschen Begriff des Interpretaments vergleichen, also mit dem, was vom Sprachzeichen durch seine Äusserung ausgelöst wird. Ein solches Interpretament kann als mentale Repräsentation gesehen werden, muss aber nicht. Dazu Gilles-Gaston Granger: "The interpretant is a commentary, a definition, a gloss on the sign in its relation to its object. The interpretant is itself symbolic expression. The sign-interpretant association...is rendered possible only by the community..." (Gaston Granger 1968; über den Unterschied Paraphrase und Sprachgebrauch siehe auch Teubert 2002).

Ein Wort wie Zwangsehe kann nur etwas Schlechtes bedeuten. Doch mit wieviel Berechtigung wird hier das pejorativ konnotierte Wort Zwangsehe verwendet? Mit wieviel Berechtigung werden hier mit dem Wort Zwangsehe alle nach islamischem Recht geschlossenen Ehen unter Generalverdacht gestellt? Dem deutschen Konzept der Zwangsehe entspricht im Englischen weithin das der arranged marriage, wenngleich es, deutlich seltener, auch das Konzept der forced marriage gibt. Den Begriff der arrangierten Ehe gibt es, allerdings recht selten, auch im Deutschen. Sind arrangierte indische oder türkische Ehen weniger glücklich als aus Liebe geschlossene Ehen? Werden in Liebesehen weniger Frauen von ihren Brüdern ermordet als in arrangierten Ehen? Sind arrangierte Ehen wirklich auf derselben Ebene wie weibliche Genitalverstümmelung? Was haben Zwangsehen mit Schwimmunterricht-Dispensen gemein? Woran erkennt man eine Zwangsehe? Wann wird eine arrangierte Ehe eine Zwangsehe? 
Etwa, wenn die Frau von ihrem Bruder ermordet wird? Sind arrangierte Ehen zu tolerieren? Dies weiss Google.de über "arrangierte ehen sind":

Arrangierte Ehen sind Zwangsheiraten und gehören verboten.

"Arrangierte Ehen sind romantisch" Indira ist 22 Jahre alt - und wurde vor einem Jahr verheiratet.

Arrangierte Ehen sind unromantisch und wenig stimulierend, aber oft sehr haltbar - eine Art EheAG.

Arrangierte Ehen sind auf manche Art erfolgreicher als westliche Ehen.

50\% arrangierte Ehen SIND Zwangsehen, ob freiwillig (dazu erzogen, zu gehorchen, sprich: konditioniert) oder nicht.

Zwangsehen und arrangierte Ehen sind in der Tat untragbar für freiheitliche Demokratien.

Arrangierte Ehen sind üblich in seinen Kreisen, und so hat auch Lord Giles nichts dagegen, die junge Lady Sophia zu heiraten.

Arrangierte Ehen sind eben Geschmackssache.

Was ist die Bedeutung von Zwangsehe und von arrangierter Ehe? Wer bestimmt über die Bedeutung? Im Mediendiskurs sind es, so scheint es, in erster Linie Zeitungstexte. Diskursbeiträge der Rezipienten des Mediendiskurses haben dagegen so gut wie keinen Einfluss. Doch sollten sie in einer demokratischen Gesellschaft gleichberechtigt sein. Das Internet, als anarchisches Medium, das in seinem Zugang praktisch egalitär ist, könnte ausgleichend wirken, wenn da nicht die Lexikographen wären, die stets schon die Autorität der Anarchie vorziehen. Denn aus den Zeitungen gelangt der Neologismus und seine Bedeutung über den Lexikographen in das Wörterbuch.

\section{Der Linguist ist kein privilegiertes Mitglied der Diskursgemeinschaft}

Der Sprachwissenschaftler, der Lexikograph, ist kein Experte für die Bedeutungen lexikalischer Einheiten. Sie können anhand von Belegen zeigen, wie Wörter gebraucht und paraphrasiert werden. Aber wir müssen von der Vorstellung Abschied nehmen, dass es die 'wahre', die 'richtige' Bedeutung gibt, und dass wir Experten haben, die uns sagen können, was Sache ist. Was eine lexikalische Einheit bedeutet, ist immer das Ergebnis einer Aushandlung zwischen den Mitgliedern einer Diskursgemeinschaft. Wie in jeder anderen Gemeinschaft lässt sich Einigkeit nicht voraussetzen. Jedes Mitglied hat das Recht, das Diskursobjekt Globalisierung so zu paraphrasieren, wie es ihm gefällt. Kann er andere nicht überzeugen und bleibt er mit seiner Einstellung allein, dann wird seine Paraphrase von anderen nicht aufgegriffen und hinterlässt in nachfolgenden Texten keine Spuren. Es liegt daher im Interesse jedes einzelnen, Wörter so zu verwenden und paraphrasieren, dass er von anderen gehört wird.

Die Aushandlung von Bedeutung ist nie abgeschlossen. Sie macht einen beträchtlichen Teil des Diskurses aus. Lexikalische Einheiten verändern ihre Bedeutung. Kein Mensch wäre vor fünfzig Jahren auf die Idee gekommen, das Diskursobjekt Abtreibung mit Mord an ungeborenen Kindern gleichzusetzen. Das Diskursobjekt Mord an ungeborenen Kindern existierte schlechthin nicht. Heute ist diese Gleichsetzung allgegenwärtig. Doch haben die Mitglieder 
der Diskursgemeinschaft immer die Möglichkeit, sie zurückzuweisen. Mit ihren Beiträgen zum Diskurs können sie Einfluss darauf nehmen, wie die Wirklichkeit des Diskurses, das heisst die Wirklichkeit, auf die wir zugreifen können, konstruiert ist. Der demokratische Diskurs ist im Prinzip ein pluralistischer Diskurs. Dass er es dem Anschein nach immer weniger ist, hängt damit zusammen, dass ein homogener Diskurs der Illusion Vorschub leistet, die Wirklichkeit des Diskurses wäre identisch mit der Welt da draussen. Viele halten die Sprache für einen Spiegel der Realität. Das Wahlverhalten von Ostdeutschen unterscheidet sich auch deshalb von dem der Westdeutschen, weil diese es nie gelernt haben, dem Mediendiskurs zu misstrauen.

Seit langem werden die öffentlichen Diskurse immer mehr entpluralisiert. Die Pressevielfalt unter der Herrschaft Wilhelm II. war ganz erheblich vielseitiger als der deutsche Zeitungsmarkt es heute ist. Das Ende der Meinungsvielfalt wird vor allem dann zur Gefahr, wenn wir, wie das heute der Fall ist, immer weniger die Gelegenheit haben, das, was uns mitgeteilt wird, an unseren eigenen Ich-Erlebnissen zu messen. Vor ein paar hundert Jahren konnten die Mitglieder einer Diskursgemeinschaft die meisten Diskursobjekte, die für sie relevant waren, in persönlicher Anschauung kennenlernen. Dass Napoleons Truppen das Land besetzten, konnte man sehen, während es dem einzelnen überlassen blieb, diese Besetzung als Befreiung oder als Besatzung zu erleben. Der öffentliche Diskurs, der seinerzeit die Bürger erheblich mehr als heute einbezog, verzeichnete beide Interpretationen. Heute jedoch ist aus dem öffentlichen Diskurs der Mediendiskurs geworden. Die einzigen Diskursbeiträge, auf die wir direkt reagieren können, sind die von Familienmitgliedern, von Freunden, Bekannten und Kollegen. Alles, was ausserhalb unserer begrenzten Lebenswelt liegt, ist uns nur über die Medien zugänglich. Wir alle mussten glauben, dass der Irak über Massenvernichtungswaffen verfügt, denn so wurde es übereinstimmend in den Medien berichtet. Es gab für uns keine Möglichkeit, diese Behauptung mit unseren eigenen Ich-Erlebnissen oder mit dem Zeugnis von den Ich-Erlebnissen von Menschen, die uns vertraut sind, zu vergleichen. Das allermeiste, was heute relevant ist, entzieht sich unserer Anschauung. Wir sind ausschliesslich auf die Medien angewiesen. Was Politiker fordern, was Experten behaupten, was das Volk denkt, weiss ich nur, soweit es von den Medien vermittelt wird. Doch sind unsere Medien den demokratischen Rechten der Diskursgemeinschaft unterworfen?

Die Medien, müssen wir annehmen, orientieren ihre Beiträge vor allem an ihrer eigenen Interessenlage. Das war der Fall in den sozialistischen Ländern. Bei gleicher Interessenlage wurde auch gleich berichtet und kommentiert. Das ist indessen auch der Fall in nichtsozialistischen Ländern. Die Interessenlage der Verantwortlichen in den Medien ist weitgehend identisch. Doch während in den sozialistischen Ländern dem öffentlichen Diskurs der oft diametral entgegengesetzte private Diskurs gegenüberstand, ist der private Diskurs in vielen westlichen Ländern, etwa in Deutschland (wenigstens in Westdeutschland), in England oder in Amerika, nur noch ein Spiegelbild des Mediendiskurses. Die Wirklichkeit des privaten Diskurses unterscheidet sich nur marginal von der Wirklichkeit, die in den Medien konstruiert wird. Das Ergebnis ist, dass der öffentlich-private Diskurs entpluralisiert wird. Alle Medien behaupten übereinstimmend, dass Globalisierung Fortschritt bedeutet. Der Einzelne hat nicht 
mehr die Möglichkeit, sich mit abweichen Paraphrasen Gehör zu verschaffen, so dass am Ende die Wirklichkeit des Mediendiskurses auch privat akzeptiert wird.

Was ist dann die Aufgabe der Linguistik? Sprachwissenschaftler haben die Mittel, den Diskurs, die Diskurse zu analysieren. Sie können deshalb leichter als andere untersuchen, wie im Diskurs Wirklichkeit konstruiert wird. Sie können daher auch die Wirklichkeiten, die in unterschiedlichen Diskursen konstruiert werden, miteinander vergleichen. (Denn es gibt sie noch, die alternativen Diskurse, beispielsweise im Internet, beispielsweise in anderen Kulturen oder in längst vergangenen Zeiten.) Ein solcher Vergleich ist die Voraussetzung dafür, dass wir uns der Relativität dieser vermittelten Wirklichkeiten bewusst werden. John Searle glaubt, dass die, die der Relativität von Wirklichkeit das Wort reden, nur einen Grund haben: "It satisfies a basic urge to power"(Searle 1998). Mir scheint dagegen, dass die, welche die von ihnen konstruierte Wirklichkeit als die Wirklichkeit schlechthin darstellen, alle anderen von ihrem demokratischen Recht, an der Aushandlung der Diskurswirklichkeit teilzuhaben, abhalten wollen.

Von unserer eng umgrenzten Lebenswelt einmal abgesehen, bleibt uns die diskursexterne Wirklichkeit unzugänglich. Alles, was sich uns präsentiert, sind diskursiv konstruierte Wirklichkeiten. Wir können uns nur dann einen eigenen Standpunkt bilden, wenn wir diese Wirklichkeiten miteinander vergleichen. Einen solchen Vergleich zu ermöglichen, das sehe ich als die Aufgabe der Sprachwissenschaft an. Es ist jedoch nicht die am Sprachsystem interessierte Linguistik, sondern die, die das, was tatsächlich gesagt wird, nennen wir es Diskurs oder parole, zu ihrem Gegenstand macht, eine Sprachwissenschaft, die sich weniger um kombinatorische Regeln und um systeminterne Oppositionen kümmert als um die Bedeutung dessen, was da gesagt ist, kurz, eine hermeneutisch verstandene Korpuslinguistik. Sie kann zum öffentlichen Diskurs einen Beitrag leisten, indem sie zeigt, welche Texte wie über welche Diskursobjekte sprechen. Sie kann ihre Interpretation davon in den Dialog der Diskursgemeinschaft einbringen. Aber welche Interpretationen sich durchsetzen und welche scheitern, sollte weder Sache der Linguisten noch derer, die über die Medien bestimmen, sein.

\section{Literaturangaben}

Allan, Graham (2000): Intertextuality. London.

Berger, Peter L./Luckmann, Thomas (1980): Die gesellschaftliche Konstruktion der Wirklichkeit. Frankfurt am Main.

Busse, Dietrich/Teubert, Wolfgang (1994): "Ist Diskurs ein sprachwissenschaftliches Objekt? Zur Methodenfrage der historischen Semantik". In: Busse, Dietrich/Hermanns, Fritz/Teubert, Wolfgang (eds.): Begriffsgeschichte und Diskursgeschichte. Opladen: 10-28. Clear, Jeremy/Fox, Gwyneth/Francis, Gill/Krishnamuthy, Ramesh/Moon, Rosamund (1996): "COBUILD: The state of the art". International Journal of Corpus Linguistics 1, 2: 303314.

Dennett, Daniel C. (1991): Consciousness Explained. Harmondsworth.

Deutsches Universalwörterbuch (1983). Mannheim. 
Fairclough, Norman (2003): Analysing Discourse: Textual Analysis for Social Research. London.

Foucault, Michel (1969): L'archeologie du savoir. Paris.

Gadamer, Hans-Georg (1965): Wahrheit und Methode. 2. Auflage. Tübingen.

Goody, Jack (1987): The Interface Between the Written and the Oral. Cambridge.

Granger, Gilles-Gaston (1968): Essai d'une philosophie du style. Paris.

Halliday, M. A. K./Teubert, Wolfgang/Yallop, Colin/Cermakova, Anna (2004): Lexicology and Corpus Linguistics: An Introduction. London.

Kristeva, Julia (1986): The Kristeva-Reader. Ed. by Toril Moi. Oxford.

Luhmann, Niklas (1998): Die Gesellschaft der Gesellschaft. 2 Bände. Frankfurt am Main.

Luria, Aleksandr Romanovich (1976): Cognitive Development: Its Cultural and Social Foundations. Ed. by Michael Cole. Cambridge, Mass.

Ong, Walter J. (1982): Orality and Literacy. London.

Ricoeur, Paul (1976): Interpretation Theory: Discourse and the Surplus of Meaning. Fort Worth, Texas.

Ricoeur, Paul (1981): Hermeneutics and the Human Sciences. Ed. and translated by John B. T. Thompson. Cambridge.

Searle, John R. (1992): The Rediscovery of Mind. Cambridge, Mass. Searle, John R. (1998): Mind, Language and Society. New York.

Teubert, Wolfgang (2002): "Die Bedeutung von Globalisierung". In: Panagl, Oswald/Stuermer, Horst (eds.): Politische Konzepte und verbale Strategien. Frankfurt: 149168.

Teubert, Wolfgang (2003): "Writing, hermeneutics and corpus linguistics". [Journal of] Logos and Language 4, 2: 1-17.

Teubert, Wolfgang (2005): "My version of corpus linguistics". International Journal of Corpus Linguistics 10/1: 1-13.

Watzlawick, Paul (1977): How Real Is Real? New York.

Wodak, Ruth (1999): The Discursive Construction of National Identity. Edinburgh. 